\title{
Optical Coherence Tomography of the Skin'
}

\author{
Julia Welzel ${ }^{\mathrm{a}}$, Eva Lankenau ${ }^{\mathrm{b}}$, Reginald Birngruber ${ }^{\mathrm{b}}$, Ralf Engelhardt ${ }^{\mathrm{b}}$ \\ ${ }^{a}$ Department of Dermatology, Medical University of Lübeck, and \\ ${ }^{\mathrm{b}}$ Medical Laser Center, Lübeck, Germany
}

Optical coherence tomography (OCT) is a new method for in vivo investigation of tissue [1, 2]. In the last few years, it has mainly been used for diagnosis of the human eye [3-6]. In contrast to this low scattering medium, the skin is a turbid, high scattering tissue. Therefore, the system needed a modification for application in dermatology [7-9]. First OCT images of human skin showed promising results [10-12].

OCT is an interferometric method which supplies informations about optical inhomogeneities of the tissue. The resolution depends mainly on the coherence length of the light source, the detection depth on the absorption and scattering of the light beam. The OCT pictures are represented as twodimensional cross-sectional images. The technique allows a noninvasive visualization of superficial layers of tissue and has a high resolution which is sufficient for detection of cell aggregates.

Our aim was to evaluate the potential role of OCT for the diagnosis of epidermal changes. Using this method we investigated anatomical and pathological structures of human skin of several locations. For the interpretation of the different structures which could be detected in the OCT images we assigned them to the skin layers by comparison with histology.

${ }^{1}$ This work was partly supported by the German Ministry of Science and Research (BMBF), FK No. 13N6302. 


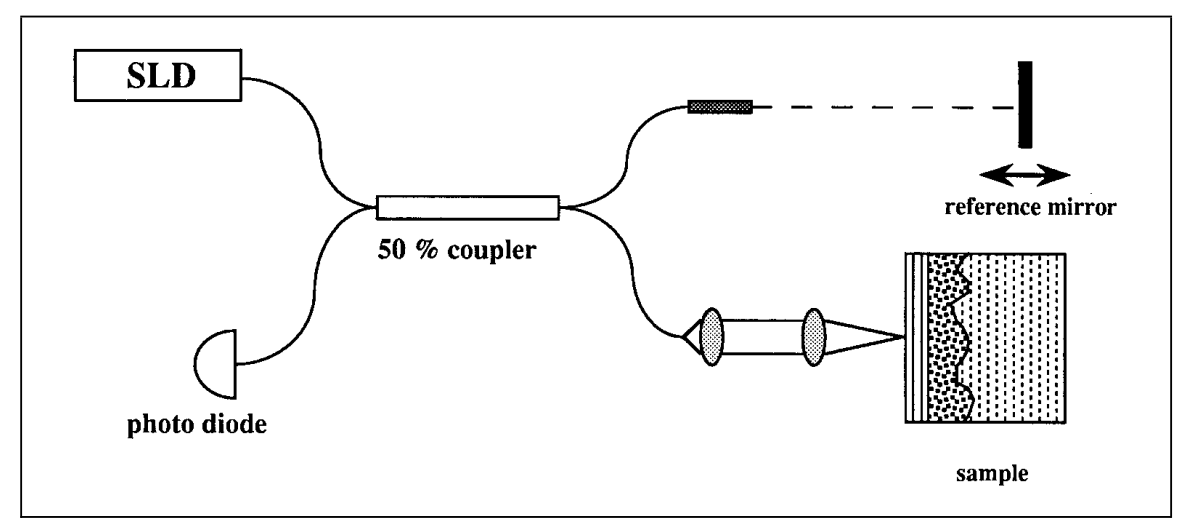

Fig. 1. Schematic diagram of the OCT system. SLD = Super luminescence diode.

\section{Method}

The OCT system is based on the principle of Michelson interferometry. The light source is an infrared superluminescence diode with a short coherence length of $15 \mu \mathrm{m}$ and a wavelength of $830 \mathrm{~nm}$. The light is coupled into an optical fiber interferometer (fig. 1). Interference occurs only when the propagation distance of the sample beam and the reference beam match within the coherence length of the light source. The optical path length distribution of the sample beam is measured by the interference modulation of the axial OCT scan. It can be interpreted as the depth-resolved reflection signal of the sample. The low coherence length of about $15 \mu \mathrm{m}$ determines the axial resolution of the system. Lateral scanning of the signal results in two-dimensional cross-sectional images which are presented in logarithmic false color or gray scales. The high dynamic range of $100 \mathrm{~dB}$ allows the detection of ultraweak light signals. Therefore, a detection depth of $0.5-1.5 \mathrm{~mm}$ can be reached depending on absorption and scattering of the signal. The images can be compared with B mode highfrequency ultrasound scans but represent optical and not acoustic inhomogeneities of the tissue. The system is described elsewhere in detail $[10,11]$.

The volunteers were seated comfortably in front of the system. The skin area of interest was positioned against an aperture of the measuring unit with mild pressure. The light beam was adjusted along the optical axis until the skin surface was brought into focus. The scan direction and length were selected depending on the size of the region of interest. Simultaneously, the OCT image was demonstrated in real time on a screen. The measuring time for one image took about $10-40 \mathrm{~s}$ depending on the desired lateral resolution and scanning length. The measurement was performed without touching the skin surface.

The OCT images were represented without image processing. The axial dimension was corrected by the average refractive index of the skin of 1.4. We investigated healthy skin of the palms and of the forearm. For interpretation of the OCT images the structures were compared with corresponding histological sections. To distinguish between the different layers seen in OCT, subepidermal blisters were induced experimentally by application of liquid nitrogen. Additionally, intraepidermal blisters of a patient with pemphigus vulgaris 

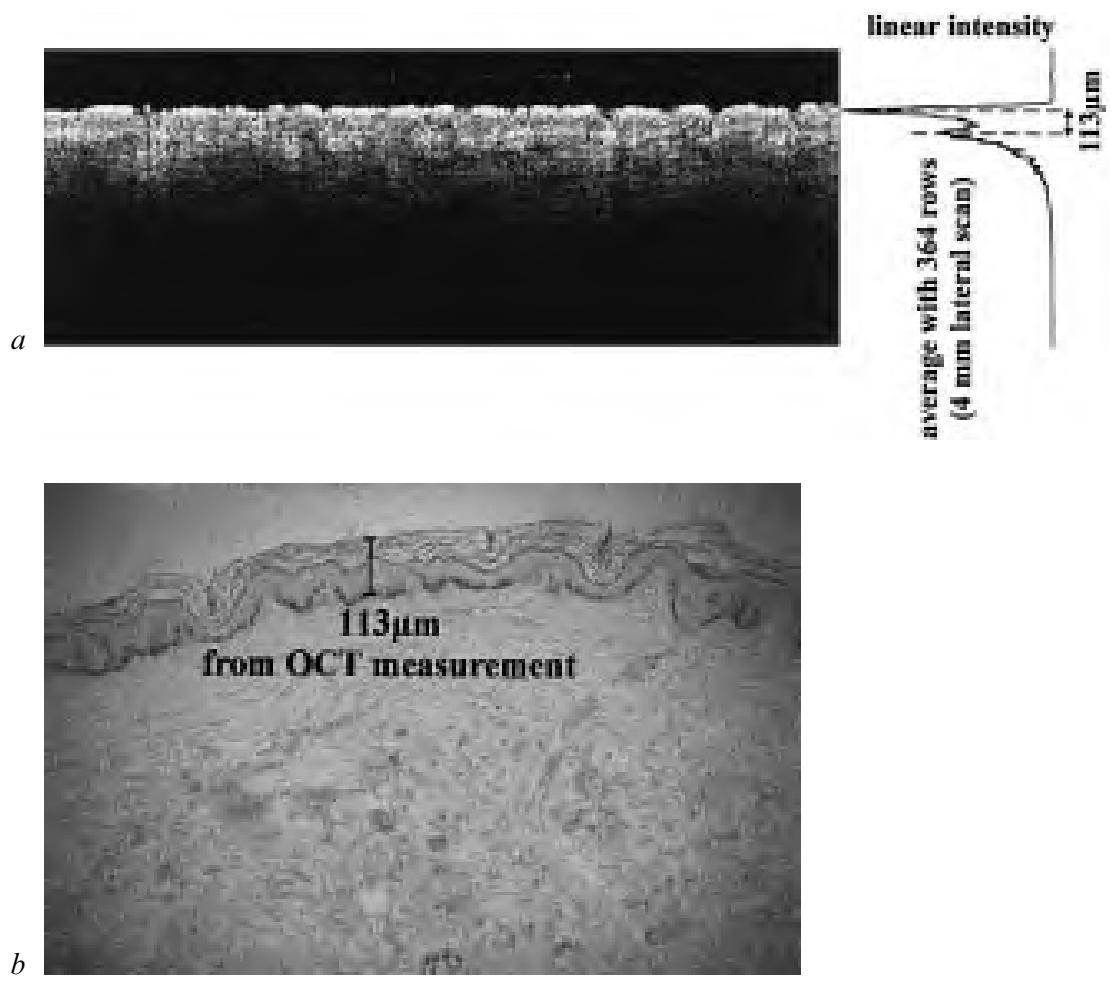

Fig. 2. Healthy skin of the forearm: OCT (a) and corresponding histology $(b)$. The superficial layer in OCT represents the entire epidermis. In the averaged A scans the intensity peak below the entrance signal corresponds to the basement membrane zone. OCT image: $4 \mathrm{~mm} \times 1.6 \mathrm{~mm}$. Histology $\times 100$.

and a mechanically induced blister on the thumb were measured. The cleft location was controlled by histology. To evaluate the potential role of OCT for diagnosis of skin tumors we investigated several melanocytic nevi and malignant melanomas. Also inflammatory skin diseases like hand eczema and scabies were measured by OCT.

\section{Results}

The OCT measurement was not noticeable by the subjects and had no side effects. The in vivo measurement could be performed in all cases without any problems.

Figure $2 \mathrm{a}$ is an example of an OCT of forearm skin. A superficial layer can be detected, bordered by a more signal-intense line. The averaged A 

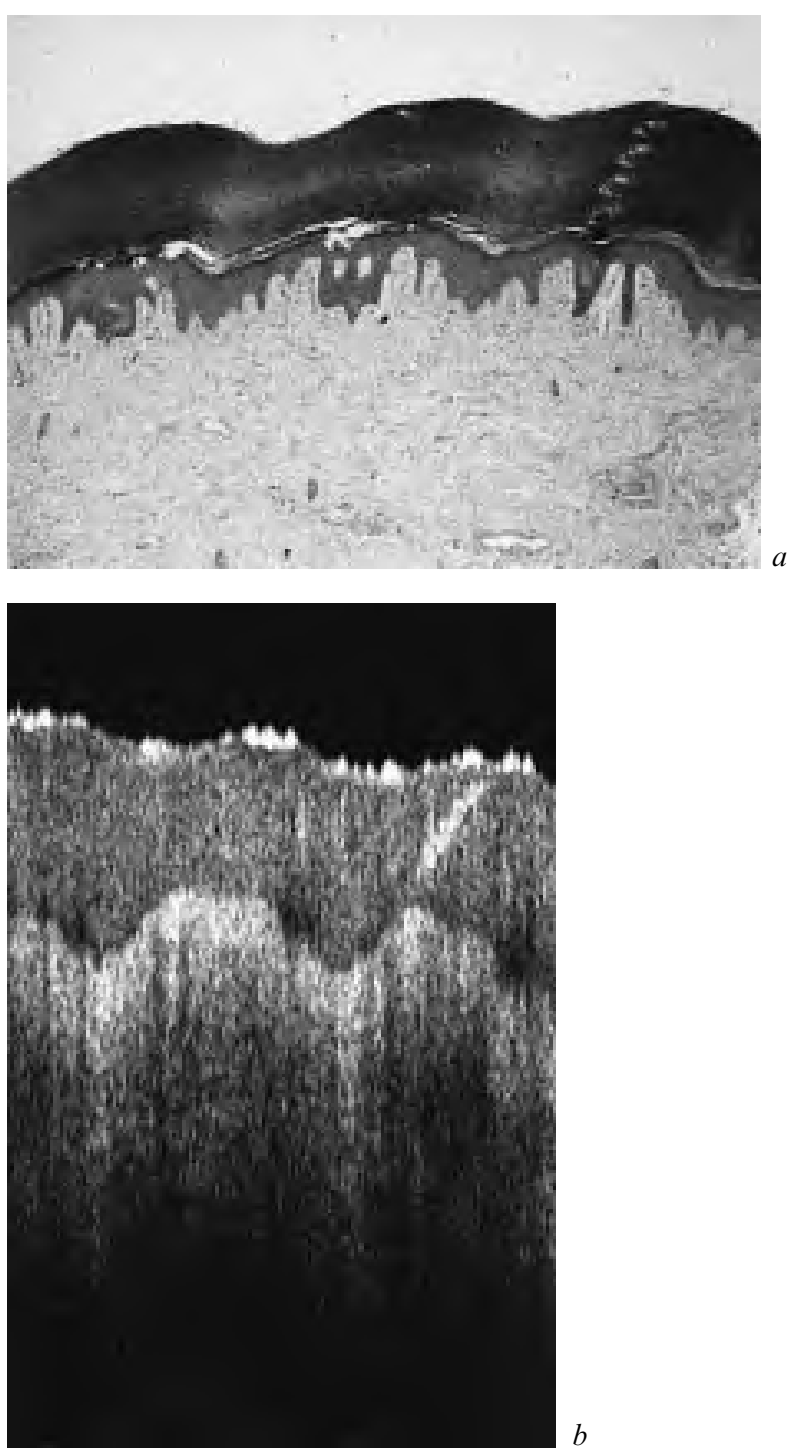

Fig. 3. Healthy skin of the fingertip: OCT (a) and corresponding histology $(b)$. The superficial transparent layer is the thick stratum corneum with a sweat gland duct. The signal-dense deeper layer can be assigned to the epidermis. OCT image: $1 \mathrm{~mm} \times 1.5 \mathrm{~mm}$. Histology $\times 100$. 


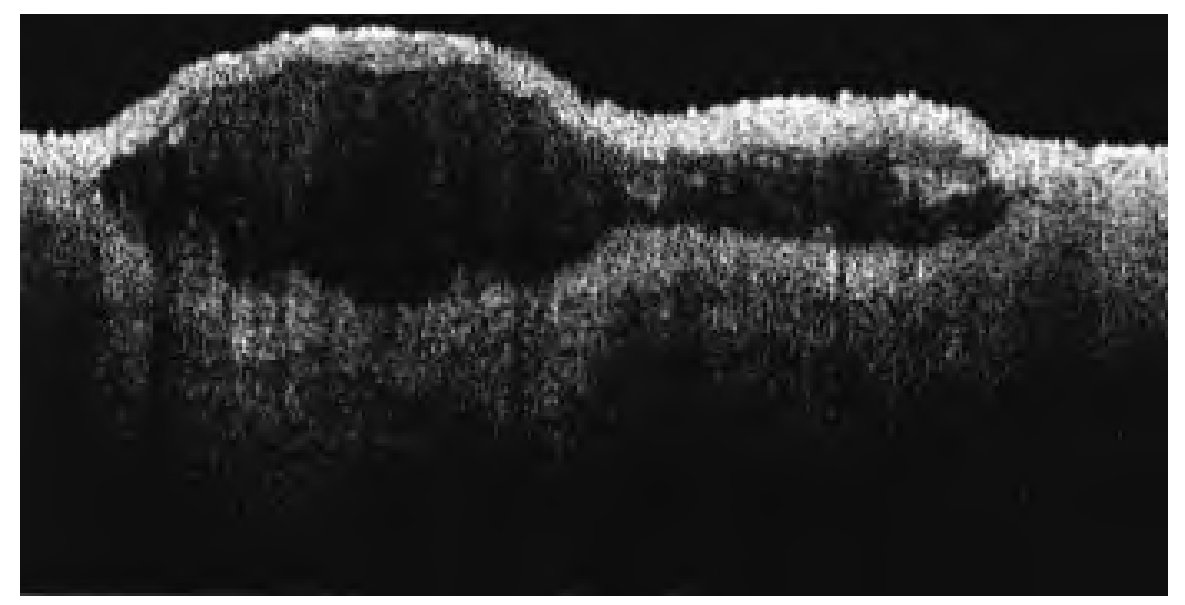

Fig. 4. OCT of a subepidermal blister on the forearm. The whole epidermis is elevated. $3 \mathrm{~mm} \times 1.5 \mathrm{~mm}$.

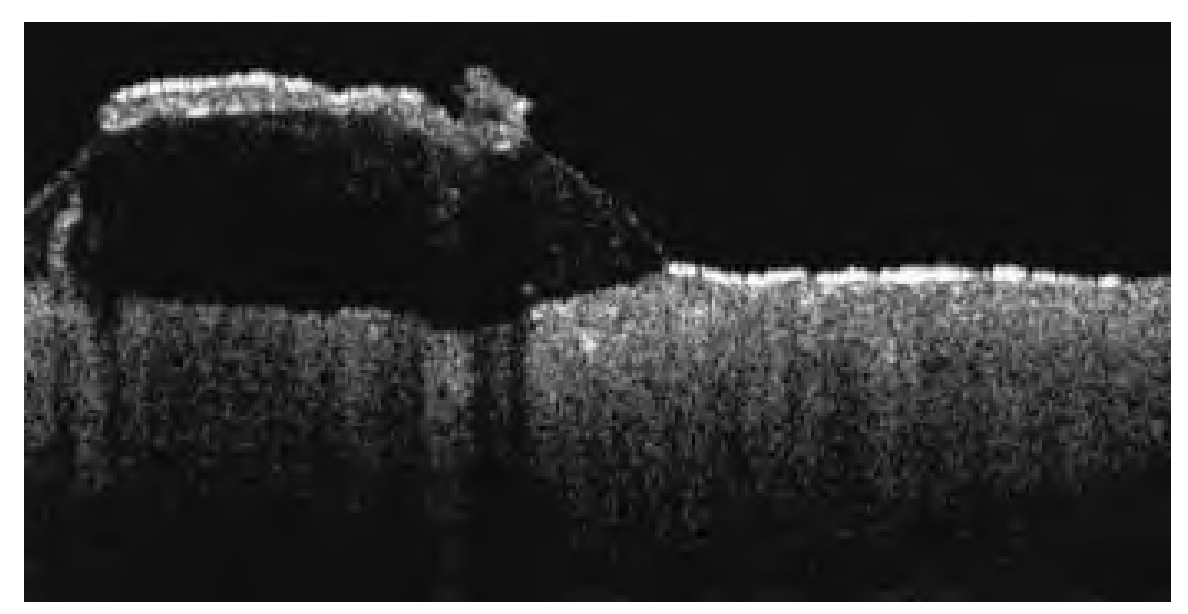

Fig. 5. OCT of an intraepidermal blister on the trunk of a patient with pemphigus vulgaris. The roof of the blister is much thinner. $2 \mathrm{~mm} \times 1.5 \mathrm{~mm}$.

scans of the image show a second intensity peak below the entrance signal corresponding to this line. The distance between these two peaks represents the thickness of the epidermis in the corresponding histological section (fig. 2b). Therefore, this superficial layer can be assigned to the epidermis and the line to the basement membrane zone. 


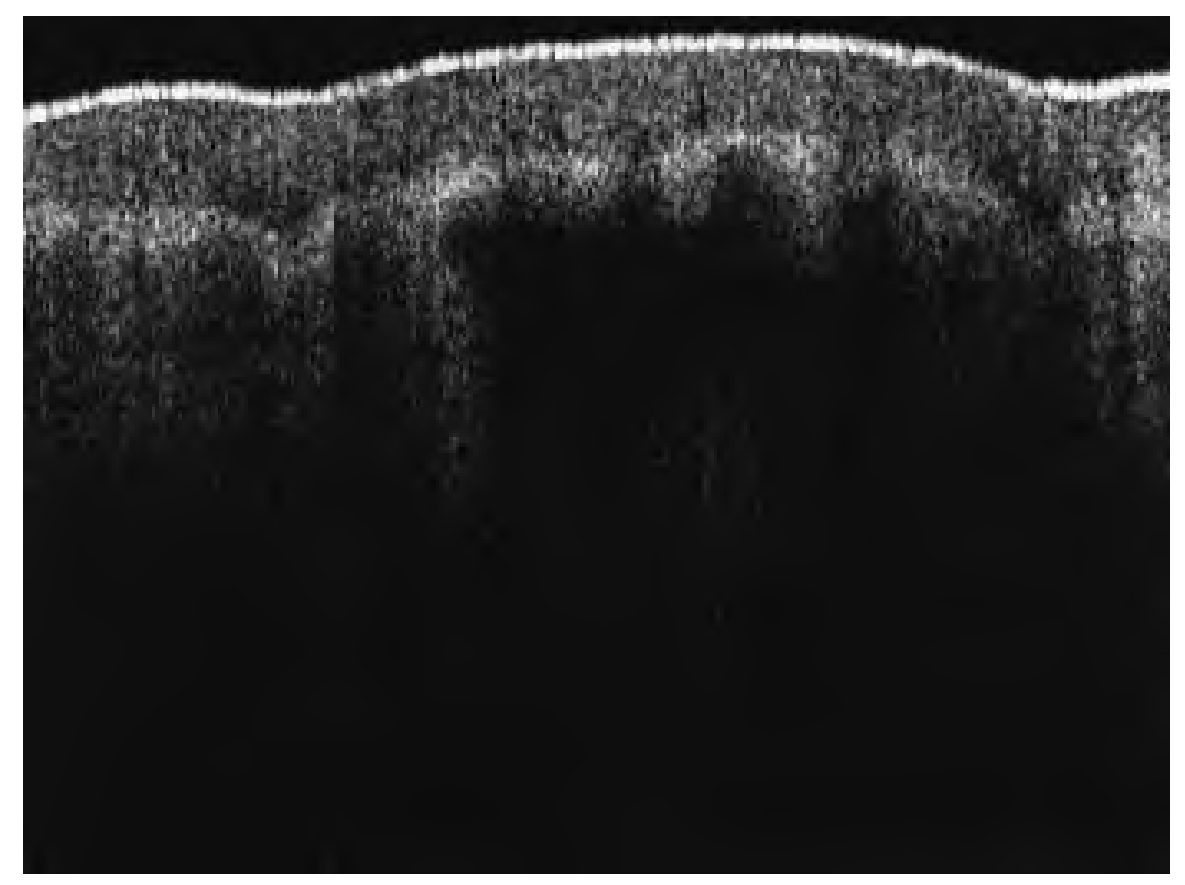

Fig. 6. OCT of an initial subepidermal blister on the palm. The blister starts below the epidermis. $2 \mathrm{~mm} \times 1.5 \mathrm{~mm}$.

On the palms and soles, the stratum corneum is much thicker. The OCT image of the skin of a fingertip exhibits two different layers (fig. 3a). The superficial one is more transparent and corresponds to the stratum corneum, the lower one to the epidermis (fig. $3 b$ ), which is more signal intense. The resolution is even high enough to demonstrate spiral sweat gland ducts in the horny layer.

A subepidermal blister on the forearm is shown in figure 4. The whole epidermis is elevated, the blister fluid is transparent. An intraepidermal blister of pemphigus vulgaris on the trunk shows a very thin roof (fig. 5). On the palm, the subepidermal blister caused by liquid nitrogen starts below the second layer which is the epidermis (fig. 6). In contrast, the mechanically induced intraepidermal blister on the thumb exhibits a cleft location below the first layer which corresponds to the thick stratum corneum (fig. 7a). The histology of the roof of the blister confirms that the cleft is located in the upper stratum spinosum (fig. $7 b$ ).

Figure 8a is an example of an OCT image of a lentigo maligna melanoma on the face. On the right side, the border to healthy skin is demonstrated 


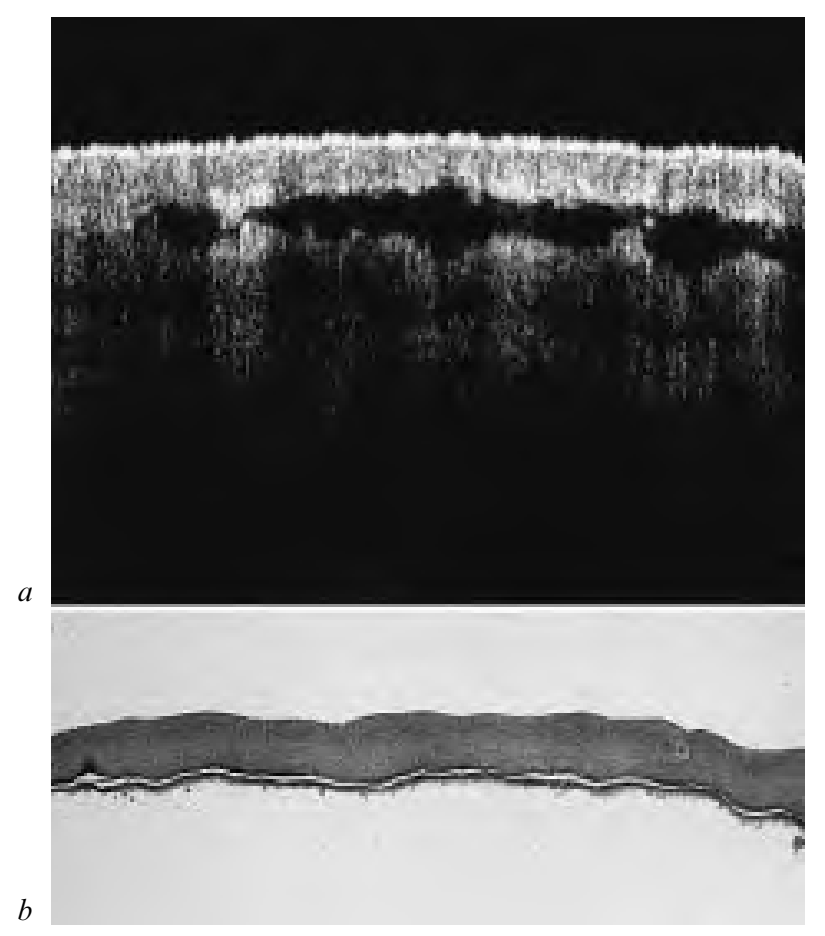

Fig. 7. An intraepidermal blister on the thumb is shown. The cleft location is below the stratum corneum in OCT $(a)$. The histology of the roof of the blister $(b)$ confirms that it contains only the stratum corneum and the stratum granulosum. OCT image: $2 \mathrm{~mm} \times 1.6 \mathrm{~mm}$. Histology $\times 100$.

with a detectable second line parallel to the entrance signal representing the basement membrane zone. This line disappears on the left side in the tumor region. On this side, irregular structures can be seen which correspond to the tumor cell aggregates in the junctional zone in the corresponding histology (fig. 8b).

The OCT image of a hand eczema (fig. 9a) shows an irregular surface with signal-poor areas in the stratum corneum and epidermis compared to the OCT image of the contralateral healthy side (fig. 9b).

Figure 10 is an OCT image of scabies. The mite can be detected in the center of the image with a burrow behind. 


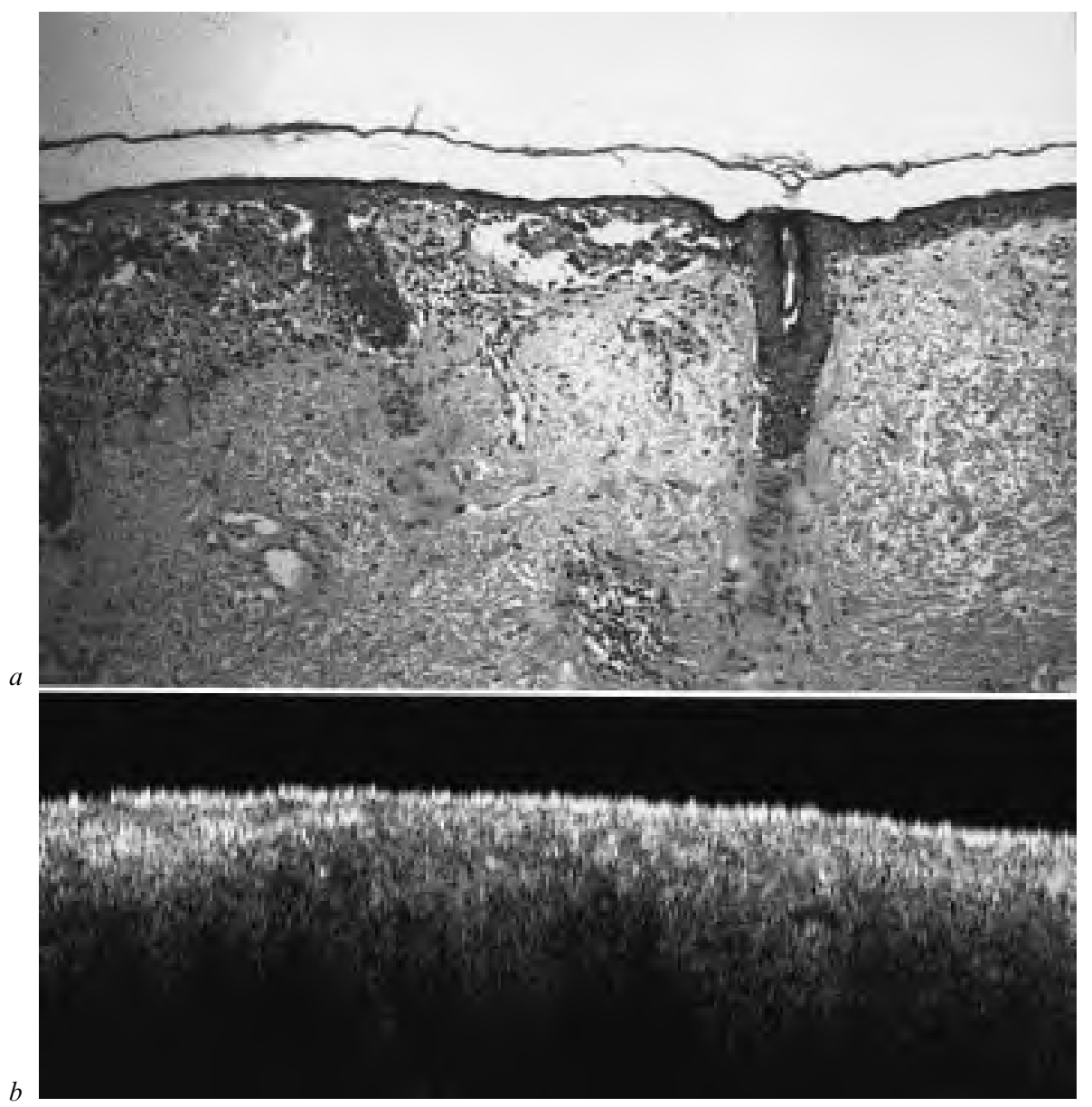

Fig. 8. OCT (a) and corresponding histology (b) of a lentigo maligna melanoma on the left side and the border to healthy skin on the right side. The melanoma nests are represented as irregular structures in OCT. OCT image: $2 \mathrm{~mm} \times 0.8 \mathrm{~mm}$. Histology $\times 200$.

\section{Discussion}

In OCT images of healthy human skin, different layers were distinguishable which could be assigned to anatomical structures seen in corresponding histological sections. On the trunk and forearm the superficial layer corresponded to the whole epidermis. An in vivo measurement of the epidermal thickness was possible. On the palms and soles the thick stratum corneum appeared as 


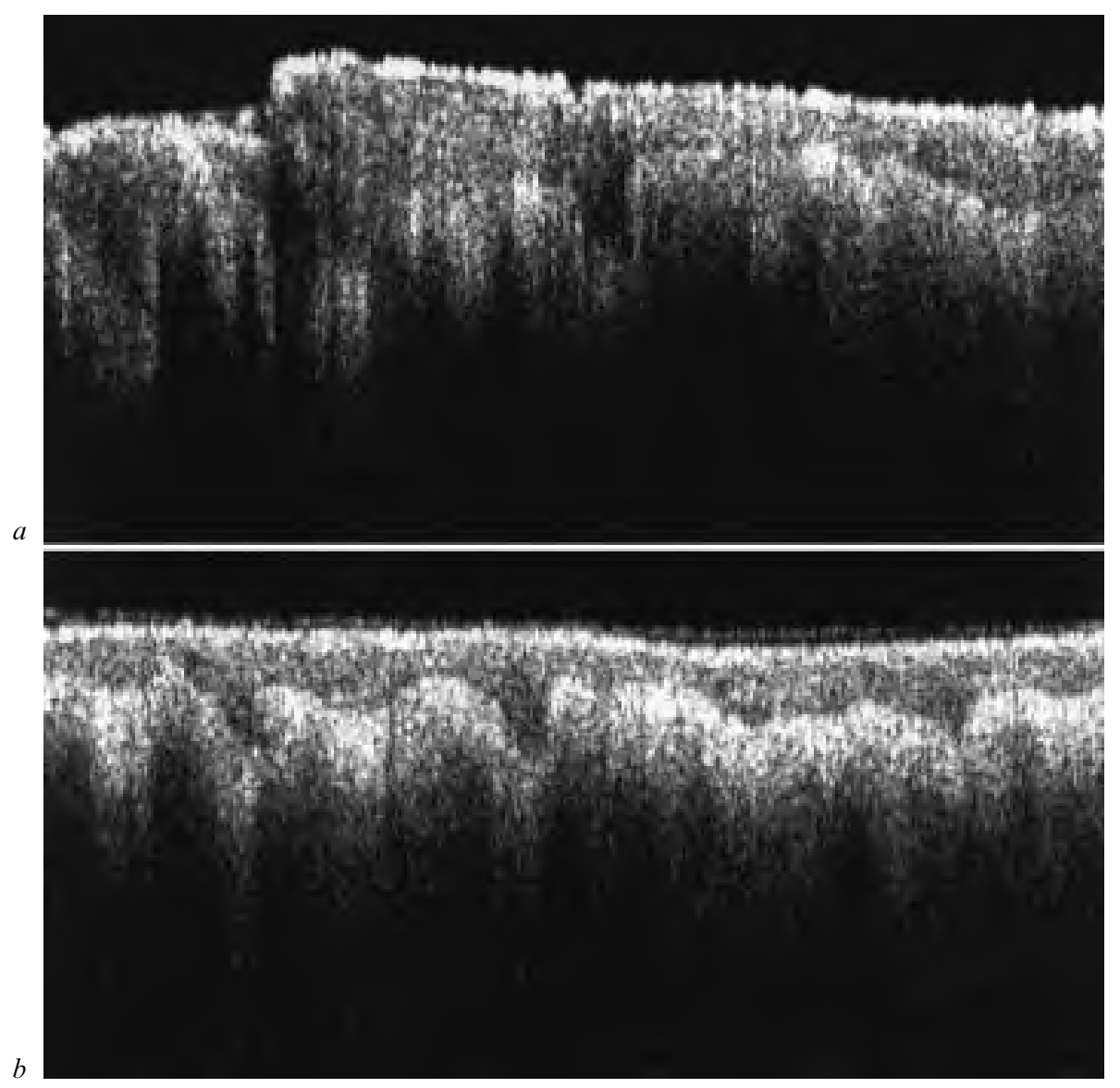

Fig. 9. OCT of a patient with hand eczema. The diseased skin (a) shows signal-poor areas compared to the unaffected skin $(b)$ of the contralateral side. $2 \mathrm{~mm} \times 1.5 \mathrm{~mm}$.

a transparent layer above the epidermis which was not detectable in other regions. The investigation of well-defined bullous changes was helpful for the interpretation of the layers seen in OCT images. The exact location of intraepidermal and subepidermal blisters was possible in all cases. A malignant melanoma was characterized in OCT with irregular structures in the junctional zone. The well-defined border between epidermis and upper dermis which was typical of healthy skin disappeared in the tumor region. In eczematous skin 


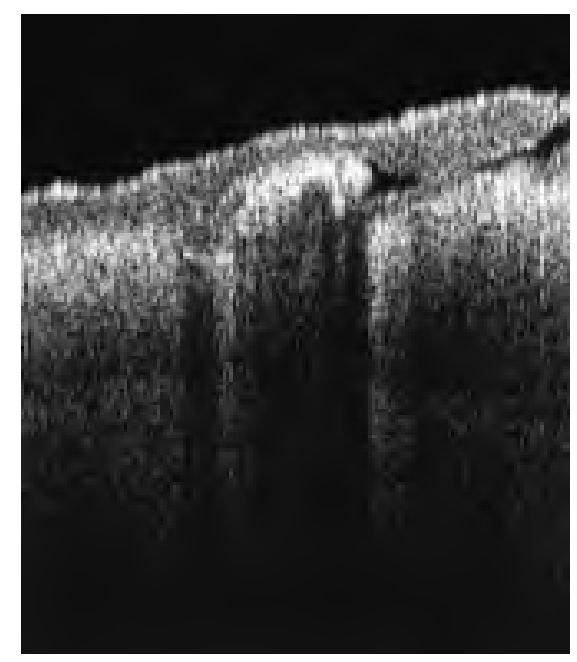

Fig. 10. OCT of a scabies mite in the center of the image with a burrow behind the mite. $1 \mathrm{~mm} \times 1.2 \mathrm{~mm}$.

signal-poor areas in the horny layer and in the epidermis could be found which might be interpreted as parakeratosis and spongiosis. A scabies mite with its burrow behind could easily be detected in the stratum corneum. In all cases, OCT corresponded well to histology.

The axial resolution of $15 \mu \mathrm{m}$ allowed the visualization of cell aggregates and layers in skin diseases and tumors. However, investigation of single cells or subcellular structures was not possible. The detection depth of OCT is generally limited by multiple scattering and attenuation of the signal. The current detection depth of about $1 \mathrm{~mm}$ was sufficient for investigation of the stratum corneum, the living epidermis and upper parts of the dermis. Thus, OCT is a promising new method for in vivo investigation of superficial skin changes. Furthermore, the technique is simple, noninvasive, practicable and fast without any side effects. OCT may be useful for noninvasive diagnosis of bullous skin diseases, skin tumors and in vivo investigation of pharmacological effects or wound healing. It might be of particular interest for the early diagnosis of malignant melanomas. Further developments will focus on real time measurements and a more flexible system including a three-dimensional scanning mode. More systematic investigations of skin lesions are necessary for the interpretation of the OCT images and the final judgement of the potential role as a diagnostic tool in dermatology. 


\section{References}

1 Huang D, Swanson EA, Lin CP, Shuman JS, Stinson WG, Chang W, Hee MR, Flotte T, Gregory K, Puliafito CA, Fujimoto JG: Optical coherence tomography. Science 1991;254:1178-1181.

2 Schmitt JM, Knüttel A, Bonner RF: Measurement of optical properties of biological tissues by low-coherence reflectometry. Appl Opt 1993;32:6032-6042.

3 Hee MR, Izatt JA, Swanson EA, Huang D, Shuman JS, Lin CP, Puliafito CA, Fujimoto JG: Optical coherence tomography of the human retina. Arch Ophthalmol 1995;113:325-332.

4 Hee MR, Puliafito CA, Wong C, Reichel E, Duker JS, Shuman JS, Swanson EA, Fujimoto JG: Optical coherence tomography of central serous chorioretinopathy. Am J Ophthalmol 1995;120: $65-74$.

5 Izatt JA, Hee MR, Swanson EA, Lin CP, Huang D, Shuman JS, Puliafito CA, Fujimoto JG: Micrometer-scale resolution imaging of the anterior eye in vivo with optical coherence tomography. Arch Ophthalmol 1994;112:1584-1589.

6 Fujimoto JG, Brezinski ME, Tearney GJ, Boppart SA, Bouma B, Hee MR, Southern JF, Swanson EA: Optical biopsy and imaging using optical coherence tomography. Nat Med 1995;1:970-972.

7 Pan Y, Birngruber R, Rosperich J, Engelhardt R: Low-coherence optical tomography in turbid tissue: Theoretical analysis. Appl Opt 1995;34:6564-6574.

8 Pan Y, Arlt S, Birngruber R, Engelhardt R: Optical coherence tomography in turbid tissue: Theoretical analysis and experimental results; in Foth HJ, Marchesini R (eds): Optical and Imaging Techniques for Biomonitoring. Proc Soc Photo Opt Instrum Eng. Washington, International Society for Optical Engineering, 1995, vol 2628, pp 239-248.

9 Pan Y, Birngruber R, Engelhardt R: Contrast limits of coherence-gated imaging in scattering media. Appl Opt 1997;36:2979-2983.

10 Lankenau E, Koch P, Engelhardt R: An imaging system for low coherence tomography; in Payne SA, Pollock CR (eds): Trends in Optics and Photonics, Volume on Advances in Optical Imaging and Photon Migration. Washington, Optical Society of America, 1996, pp 247-249.

11 Pan Y, Lankenau E, Welzel J, Birngruber R, Engelhardt R: Optical coherence gated imaging of biological tissues. IEEE J Selected Top Quantum Electronics Lasers Med Biol 1996;2:1029-1034.

12 Schmitt JM, Yadlowsky MJ, Bonner RF: Subsurface imaging of living skin with optical coherence microscopy. Dermatology 1995;191:93-98.

Dr. med. Julia Welzel, Department of Dermatology, Medical University of Lübeck, Ratzeburger Allee 160, D-23538 Lübeck (Germany) 